\title{
Subgraph densities in signed graphons and the local Simonovits-Sidorenko conjecture
}

\author{
László Lovász* \\ Institute of Mathematics, Eötvös Loránd University \\ Budapest, Hungary
}

Submitted: Feb 17, 2011; Accepted: Jun 6, 2011; Published: Jun 14, 2011

Mathematics Subject Classification: 05C35

\begin{abstract}
We prove inequalities between the densities of various bipartite subgraphs in signed graphs. One of the main inequalities is that the density of any bipartite graph with girth $2 r$ cannot exceed the density of the $2 r$-cycle.

This study is motivated by the Simonovits-Sidorenko conjecture, which states that the density of a bipartite graph $F$ with $m$ edges in any graph $G$ is at least the $m$-th power of the edge density of $G$. Another way of stating this is that the graph $G$ with given edge density minimizing the number of copies of $F$ is, asymptotically, a random graph. We prove that this is true locally, i.e., for graphs $G$ that are "close" to a random graph.

Both kinds of results are treated in the framework of graphons (2-variable functions serving as limit objects for graph sequences), which in this context was already used by Sidorenko.
\end{abstract}

\footnotetext{
${ }^{*}$ Research supported by ERC Grant No. 227701.
} 


\section{Contents}

1 Introduction $\quad 2$

2 Preliminaries 4

2.1 Notation . . . . . . . . . . . . . . . . . . . . . 4

2.2 Kernel operators and their norms . . . . . . . . . . . . . . . . . . . 4

3 Density inequalities for signed graphons $\quad 6$

3.1 Ordering signed graphons . . . . . . . . . . . . . . . . . . . . . 6

3.2 A generalized Cauchv-Schwarz inequality . . . . . . . . . . . . . . . 7

3.3 Inequalities between densities . . . . . . . . . . . . . . . . . . 8

3.4 Special graphs and examples . . . . . . . . . . . . . . . . . . . . 10

3.5 The main inequalities between graphs . . . . . . . . . . . . . . . . . . 13

4 Local Sidorenko Conjecture $\quad 18$

$\begin{array}{lll}5 & \text { Variations } & 19\end{array}$

\section{Introduction}

Let $F$ be a bipartite graph with $k$ nodes and $l$ edges and let $G$ be any graph with $n$ nodes and $m=p\left(\begin{array}{l}n \\ 2\end{array}\right)$ edges. Simonovits [3, 10] conjectured that the number of copies of $F$ in $G$ is at least $p^{l}\left(\begin{array}{l}n \\ k\end{array}\right)+o\left(p^{l} n^{k}\right)$ (where we consider $k$ and $l$ fixed, and $\left.n \rightarrow \infty\right)$.

Sidorenko [7, 8, 9] conjectured a stronger exact inequality. To state this formulation, we count homomorphisms instead of copies of $F$. Let hom $(F, G)$ denote the number of homomorphisms from $F$ into $G$. Since we need this notion for the case when $F$ and $G$ are multigraphs, we count here pairs of maps $\phi: V(F) \rightarrow V(G)$ and $E(F) \rightarrow E(G)$ such that incidence is preserved: if $i \in V(F)$ is incident with $e \in E(F)$, then $\phi(i)$ is incident with $\psi(e)$. We will also consider the normalized version $t(F, G)=\operatorname{hom}(F, G) / n^{k}$. If $F$ and $G$ are simple, then $t(F, G)$ is the probability that a random map $\phi: V(F) \rightarrow V(G)$ preserves adjacency. We call this quantity the density of $F$ in $G$.

In this language, the conjecture says that for any bigraph $F$ and any graph $G$,

$$
t(F, G) \geq t\left(K_{2}, G\right)^{|E(F)|}
$$

(this is an exact inequality with no error terms). We can formulate this as an extremal result in two ways: First, for every graph $G$, among all bipartite graphs with a given number of edges, it is the graph consisting of disjoint edges (the matching) that has the smallest density in $G$. Second, for every bipartite graph $F$, among all graphs on $n$ nodes and edge density $p$, the random graph $\mathbb{G}(n, p)$ has the smallest density of $F$ in it (asymptotically, with large probability). 
Sidorenko proved his conjecture in a number of special cases: for trees $F$, and also for bigraphs $F$ where one of the color classes has at most 4 nodes. Since then, the only substantial progress was that Hatami [4 proved the conjecture for cubes, and Conlon, Fox and Sudakov [2] proved it for bigraphs having a node connected to all nodes on the other side.

Sidorenko gave an analytic formulation of this conjecture, which we will use. Let $F$ be a bipartite multigraph with a bipartition $(A, B)$; if we say that $i j \in E(F)$, we assume that the labeling is such that $i \in A$ and $j \in B$. Assign a real variable $x_{i}$ to each $i \in A$ and a real variable $y_{j}$ to each $j \in B$. Let $W:[0,1]^{2} \rightarrow \mathbb{R}_{+}$be a bounded measurable function, and define

$$
t(F, W)=\int_{[0,1]^{V(F)}} \prod_{i j \in E(F)} W\left(x_{i}, y_{j}\right) \prod_{i \in A} d x_{i} \prod_{j \in B} d y_{j} .
$$

Every graph $G$ can be represented by a function $W_{G}$ : Let $V(G)=\{1, \ldots, n\}$. Split the interval $[0,1]$ into $n$ equal intervals $J_{1}, \ldots, J_{n}$, and for $x \in J_{i}, y \in J_{j}$ define $W_{G}(x, y)=$ $\mathbb{1}_{i j \in E(G)}$. (The function obtained this way is symmetric.) Then we have

$$
t(F, G)=t\left(F, W_{G}\right) .
$$

Note, however, that definition (2) makes sense without assuming that $W$ is symmetric.

In this analytic language, the conjecture says that for every bipartite graph $F$ and bounded measurable function $W:[0,1]^{2} \rightarrow \mathbb{R}_{+}$, we have

$$
t(F, W) \geq t\left(K_{2}, W\right)^{|E(F)|} .
$$

Since both sides are homogeneous in $W$ of the same degree, we can scale $W$ and assume that

$$
t\left(K_{2}, W\right)=\int_{[0,1]^{2}} W(x, y) d x d y=1 .
$$

Then we want to conclude that $t(F, W) \geq 1$. In other words, the function $W \equiv 1$ minimizes $t(F, W)$ among all functions $W \geq 0$ with $\int W=1$.

The goal of this paper is to prove that this holds locally, i.e., for functions $W$ sufficiently close to 1 . Most of the time we will work with the function $U=W-1$, which can take negative values. Most of our work will concern estimates for the values $t\left(F^{\prime}, U\right)$ for various (bipartite) graphs $F^{\prime}$. This type of question seems to have some interest on its own, because it can be considered as an extension of extremal graph theory to signed graphs. 


\section{Preliminaries}

\section{$2.1 \quad$ Notation}

A bigraph will mean a bipartite multigraph with a fixed bipartition, in which a first and second bipartition class is specified. So the complete bigraphs $K_{a, b}$ and $K_{b, a}$ are different.

We have to consider graphs that are partially labeled. More precisely, a $k$-labeled graph $F$ has a subset $S \subseteq V(F)$ of $k$ elements labeled $1, \ldots, k$ (it can have any number of unlabeled nodes). For some basic graphs, it is good to introduce notation for some of their labeled versions. Let $P_{n}$ denote the unlabeled path with $n$ nodes (so, with $n-1$ edges). Let $P_{n}^{\bullet}$ denote the path $P_{n}$ with one of its endpoints labeled. Let $P_{n}^{\bullet \bullet}$ denote the $P_{n}$ with both of its endpoints labeled. Let $C_{n}$ denote the unlabeled cycle with $n$ nodes, and let $C_{n}^{\bullet}$ be this cycle with one of its nodes labeled. Let $K_{a, b}$ denote the unlabeled complete bigraph; let $K_{a, b}^{\bullet}$ denote the complete bigraph with its first bipartition class labeled. Note that $K_{2,2} \cong C_{4}$, but $K_{2,2}^{\bullet}$ and $C_{4}^{\bullet}$ are different as partially labeled graphs.

We extend the definition of subgraph densities to $k$-labeled graphs. Let $F$ be a graph on node set $[n]$, of which nodes $1, \ldots, k$ are considered as labeled. For given $x_{1}, \ldots, x_{k} \in I$, we define

$$
t_{x_{1} \ldots x_{k}}(F, W)=\int_{[0,1]^{n-k}} \prod_{i j \in E(F)} W\left(x_{i}, x_{j}\right) d x_{k+1} \ldots d x_{n}
$$

(this is a function of $x_{1}, \ldots, x_{k}$ ).

The most important use of partial labeling is to define a product: if $F$ and $G$ are $k$-labeled graphs, then $F G$ denotes the $k$-labeled graph obtained by taking their disjoint union and identifying nodes with the same label. For a $k$-labeled graph $F,[[F]]$ denotes the graph obtained by unlabeling all nodes. The graph $O_{k}$ with $k$ labeled nodes, no unlabeled nodes and no edges is a unit element: $O_{k} F=F$ for every $k$-labeled graph $F$.

\subsection{Kernel operators and their norms}

We set $I=[0,1]$. Let $\mathcal{W}$ denote the set of bounded measurable functions $U: I^{2} \rightarrow \mathbb{R}$; $\mathcal{W}_{+}$is the set of bounded measurable functions $U: I^{2} \rightarrow \mathbb{R}_{+}$, and $\mathcal{W}_{1}$ is the set of measurable functions $U: I^{2} \rightarrow[-1,1]$. Every function $U \in \mathcal{W}$ defines a kernel operator $L_{1}(f) \rightarrow L_{1}(f)$ by

$$
f \mapsto \int_{I} U(., y) f(y) d y
$$

For $U, W \in \mathcal{W}$, we denote by $U \circ W$ the function

$$
(U \circ W)(x, y)=\int_{I} U(x, z) W(z, y) d z
$$


(this corresponds to the product of $U$ and $W$ as kernel operators). For every $W \in \mathcal{W}$, we denote by $W^{\top}$ the function obtained by interchanging the variables in $W$.

We will also need the tensor product $U \otimes W$ of two functions $U, W \in \mathcal{W}$; this is defined as a function $I^{2} \times I^{2} \rightarrow \mathbb{R}$ by

$$
(U \otimes W)\left(x_{1}, x_{2}, y_{1}, y_{2}\right)=U\left(x_{1}, y_{1}\right) W\left(x_{2}, y_{2}\right)
$$

This function is not in $\mathcal{W}$; however, we can consider any measure preserving map $\varphi: I \rightarrow$ $I^{2}$, and define the function

$$
(U \otimes W)^{\varphi}(x, y)=(U \otimes W)(\varphi(x), \varphi(y))
$$

It does not really matter which particular measure preserving map we use here: these functions obtained from different maps $\phi$ have the same subgraph densities. In fact, we have

$$
t\left(F,(U \otimes W)^{\phi}\right)=t(F, U \otimes W)=t(F, U) t(F, W)
$$

for every graph $F$. We will call any of the functions $(U \otimes W)^{\phi}$ the tensor product of $U$ and $W$.

We consider various norms on the space $\mathcal{W}$. We need the standard $L_{2}$ and $L_{\infty}$ norms

$$
\|U\|_{2}=\left(\int_{I^{2}} U(x, y)^{2} d x d y\right)^{1 / 2}, \quad\|U\|_{\infty}=\sup \operatorname{ess}|U(x, y)| .
$$

For graph theory, the cut norm is very useful:

$$
\|U\|_{\square}=\sup _{S, T \subseteq I}\left|\int_{S \times T} U(x, y) d x d y\right| .
$$

This norm is only a factor of at most 4 away from the operator norm of $U$ as a kernel operator $L_{\infty}(I) \rightarrow L_{1}(I)$.

The functional $t(F, U)$ gives rise to further useful norms. It is trivial that $t\left(C_{2}, U\right)^{1 / 2}=$ $\|U\|_{2}$. The value $t\left(C_{2 r}, U\right)^{1 /(2 r)}$ is the $r$-th Schatten norm of the kernel operator defined by $U$. It was proved in [1] that it is closely related to the cut norm: for $U \in \mathcal{W}_{1}$,

$$
\|U\|_{\square}^{4} \leq t\left(C_{4}, U\right) \leq 4\|U\|_{\square}
$$

The other Schatten norms also define the same topology on $\mathcal{W}_{1}$ as the cut norm (cf. Corollary 3.12).

It is a natural question for which graphs does $t(F, W)^{1 /|E(F)|}$ or $t(F,|W|)^{1 /|E(F)|}$ define a norm on $\mathcal{W}$. Besides even cycles and complete bigraphs, a remarkable class was found by Hatami [4]: he proved that $t(F,|W|)^{1 /|E(F)|}$ is a norm if $F$ is a cube. He also proved the fact (attributed to B. Szegedy) that Sidorenko's conjecture is true whenever $F$ is such a "norming" graph. However, a characterization of such graphs is open. 


\section{Density inequalities for signed graphons}

\subsection{Ordering signed graphons}

For two bigraphs $F$ and $G$, we say that $F \leq G$ if $t(F, U) \leq t(G, U)$ for all $U \in \mathcal{W}_{1}$. We say that $G \geq 0$ if $t(G, U) \geq 0$ for all $U \in \mathcal{W}_{1}$. Note that if $U$ is nonnegative, then trivially $G \subseteq F$ implies that $t(F, U) \leq t(G, U)$; but since we allow negative values, such an implication does not hold in general. For example, $F \geq 0$ cannot hold for any bigraph $F$ with an odd number of edges, since then $t(F,-U)=-t(F, U)$.

The ordering is a bit counterintuitive since larger graphs tend to be smaller in the ordering. For example, $t(F, U) \leq 1=t\left(K_{0}, U\right)=t\left(K_{1}, U\right)$ for every $U$, so $F \leq K_{1}$ and $F \leq K_{0}$ for any bigraph $F$ (here $K_{1}$ may have its single node either in its first or second color class, and $K_{0}$ is the empty graph). Lemmas 3.9 and 3.15 provide other examples.

We start with some simple facts about this partial order on graphs.

Proposition 3.1 If $F$ and $G$ are nonisomorphic bigraphs without isolated nodes such that $F \leq G$, then $|E(F)| \geq|E(G)|,|E(G)|$ is even, and $G \geq 0$. Furthermore, $|t(F, U)| \leq$ $t(G, U)$ for all $U \in \mathcal{W}_{1}$.

The proof of this is based on a technical lemma, which is close to facts that are well known, but not in the exact form needed here.

Lemma 3.2 Let $F$ and $G$ be nonisomorphic bigraphs without isolated nodes. Then for every $U \in \mathcal{W}_{1}$ and $\varepsilon>0$ there exists a function $U^{\prime} \in \mathcal{W}_{1}$ such that $\left\|U-U^{\prime}\right\|_{\infty}<\varepsilon$ and $t\left(F, U^{\prime}\right) \neq t\left(G, U^{\prime}\right)$.

A similar assertion (with a similar proof) holds in the context of non-bipartite graphs as well.

Proof. First we show that if $F$ and $G$ are two bigraphs without isolated nodes such that $t(F, W)=t(G, W)$ for every $W \in \mathcal{W}_{1}$, then $F \cong G$. Consider the function $U=\mathbb{1}_{x, y \leq 1 / 2}$. Then $t(F, U)=2^{-|V(F)|}$, so $t(F, U)=t(G, U)$ implies that $|V(F)|=|V(G)|$. Using the function $U \equiv 1 / 2$, we get similarly that $|E(F)|=|E(G)|$. Using this, we get (by scaling $W)$ that $t(F, W)=t(G, W)$ for every $W \in \mathcal{W}$.

For every multigraph $H$ we have

$$
t(F, H)=t\left(F, W_{H}\right)=t\left(G, W_{H}\right)=t(G, H),
$$

and hence it follows that

$$
\operatorname{hom}(F, H)=t(F, H)|V(H)|^{|V(F)|}=t(G, H)|V(G)|^{|V(F)|}=\operatorname{hom}(G, H) .
$$

From this it follows by standard arguments that $F \cong G$ (e.g., we can apply Theorem 1 (iii) of [5] to the 2-partite structures $(V, E, J)$, where $G=(V, E)$ is a multigraph and $J$ is the incidence relation between nodes and edges). 
Since $F$ and $G$ are non-isomorphic, this argument shows that there exists a function $W \in \mathcal{W}_{1}$ such that $t(F, W) \neq t(G, W)$. The values $t(F,(1-s) U+s W)$ and $t(F,(1-s) U+$ $s W)$ are polynomials in $s$ that differ for $s=1$. Therefore, there is a value $0 \leq s \leq \varepsilon$ for which they differ. Since $(1-s) U+s W \in \mathcal{W}_{1}$ and $\|U-((1-s) U+s W)\|_{\infty}=s\|U-W\|_{\infty} \leq$ $\varepsilon$, this proves the lemma.

Proof of Proposition 3.1. Applying the definition of $F \leq G$ with $U=1 / 2$, we get that $2^{-|E(F)|} \leq 2^{-|E(G)|}$, and hence $|E(F)| \geq|E(G)|$. The relation $F \leq G$ implies that $t(F, U)^{2}=t(F, U \otimes U) \leq t(G, U \otimes U)=t(G, U)^{2}$ also holds, so $|t(F, U)| \leq|t(G, U)|$ for all $U \in \mathcal{W}_{1}$. By Lemma 3.2. $U$ can be perturbed by arbitrarily little to get a $U^{\prime} \in \mathcal{W}_{1}$ with $t\left(F, U^{\prime}\right) \neq t\left(G, U^{\prime}\right)$, then $t\left(F, U^{\prime}\right)<t\left(G, U^{\prime}\right)$ and $\left|t\left(F, U^{\prime}\right)\right| \leq\left|t\left(G, U^{\prime}\right)\right|$ imply that $t\left(G, U^{\prime}\right)>0$. Since $U^{\prime}$ is arbitrarily close to $U$, this implies that $t(G, U) \geq 0$, and so $G \geq 0$. Since this holds for $U$ replaced by $-U$, it follows that $G$ must have an even number of edges.

\subsection{A generalized Cauchy-Schwarz inequality}

We need the following generalization of the Cauchy-Schwarz inequality:

Lemma 3.3 Let $f_{1}, \ldots, f_{n}: I^{k} \rightarrow \mathbb{R}$ be bounded measurable functions, and suppose that for each variable there are at most two functions $f_{i}$ that depend on that variable. Then

$$
\int_{I^{k}} f_{1} \ldots f_{n} \leq\left\|f_{1}\right\|_{2} \ldots\left\|f_{n}\right\|_{2} .
$$

This will follow from an inequality concerning a statistical physics type model. Let $G=(V, E)$ be a multigraph (without loops), and for each $i \in V$, let $f_{i} \in L_{2}\left(I^{E}\right)$ be such that $f_{i}$ depends only on the variables $x_{j}$ where edge $j$ is incident with node $i$. Let $f=\left(f_{i}: i \in V\right)$, and define

$$
\operatorname{tr}(G, f)=\int_{I^{E}} \prod_{i \in V} f_{i}(x) d x
$$

(where the variables corresponding to the edges not incident with $i$ are dummies in $f_{i}$ ).

Lemma 3.4 For every multigraph $G$ and assignment of functions $f$,

$$
\operatorname{tr}(G, f) \leq \prod_{i \in V}\left\|f_{i}\right\|_{2} .
$$

Proof. By induction on the chromatic number of $G$. Let $V_{1}, \ldots, V_{r}$ be the color classes of an optimal coloring of $G$. Let $S_{1}=V_{1} \cup \cdots \cup V_{\lfloor r / 2\rfloor}$ and $S_{2}=V \backslash S_{1}$. Let $E_{0}$ be the set 
of edges between $S_{1}$ and $S_{2}$, and let $E_{i}$ be the set of edges induced by $S_{i}$. Let $x_{i}$ be the vector formed by the variables in $E_{i}$. Then

$$
\operatorname{tr}(G, f)=\int_{I^{E_{0}}}\left(\int_{I^{E_{1}}} \prod_{i \in S_{1}} f_{i}(x) d x_{1}\right)\left(\int_{I^{E_{2}}} \prod_{i \in S_{2}} f_{i}(x) d x_{2}\right) d x_{0} .
$$

The outer integral can be estimated using the Cauchy-Schwarz inequality:

$$
\operatorname{tr}(G, f)^{2} \leq \int_{I^{E_{0}}}\left(\int_{I^{E_{1}}} \prod_{i \in S_{1}} f_{i}(x) d x_{1}\right)^{2} d x_{0} \int_{I^{E_{0}}}\left(\int_{I^{E_{2}}} \prod_{i \in S_{2}} f_{i}(x) d x_{2}\right)^{2} d x_{0} .
$$

Let $G_{1}$ be defined as the graph obtained by taking a disjoint copy $\left(S_{1}^{\prime}, E_{1}^{\prime}\right)$ of the graph $\left(S_{1}, E_{1}\right)$, and connecting each node $i \in S_{1}$ to the corresponding node $i^{\prime} \in S_{1}^{\prime}$ by as many edges as those joining $i$ to $S_{2}$ is $G$. Note that these newly added edges correspond to the edges of $E_{0}$ in a natural way. We assign to each node the same function as before, and also the same function (with differently named variables for the edges in $E_{1}^{\prime}$ ) to $i^{\prime}$. Then the first factor in (6) can be written as

$$
\iint_{I^{E_{0}}} \int_{I^{E_{1}}} \int_{I^{E_{1}^{\prime}}} \prod_{i \in S_{1} \cup S_{1}^{\prime}} f_{i}(x) d x_{1} d x_{0}=\operatorname{tr}\left(G_{1}, f\right) .
$$

We define $G_{2}$ analogously, and get that the second factor in (6) is just $\operatorname{tr}\left(G_{2}, f\right)$. So we have

$$
\operatorname{tr}(G, f)^{2} \leq \operatorname{tr}\left(G_{1}, f\right) \operatorname{tr}\left(G_{2}, f\right)
$$

Next we remark that for $r>2$, the graphs $G_{1}$ and $G_{2}$ have chromatic number at most $\lceil r / 2\rceil<r$, and so we can apply induction and use that

$$
\operatorname{tr}\left(G_{j}, f\right) \leq \prod_{i \in V\left(G_{j}\right)}\left\|f_{i}\right\|_{2}=\prod_{i \in S_{j}}\left\|f_{i}\right\|_{2}^{2}
$$

If $r=2$, then $G_{j}$ has edges connecting pairs $i, i^{\prime}$ only, and so

$$
\operatorname{tr}\left(G_{j}, f\right)=\prod_{i \in S_{j}}\left\|f_{i}\right\|_{2}^{2}
$$

In both cases, the inequality in the lemma follows by (7).

\subsection{Inequalities between densities}

Let $F_{1}$ and $F_{2}$ be two $k$-labeled graphs. Then the Cauchy-Schwarz inequality implies that for all $U \in \mathcal{W}$

$$
t\left(\left[\left[F_{1} F_{2}\right]\right], U\right)^{2} \leq t\left(\left[\left[F_{1}^{2}\right]\right], U\right) t\left(\left[\left[F_{2}^{2}\right]\right], U\right) .
$$


With the notation introduced above, this can be written as

$$
\left[\left[F_{1} F_{2}\right]\right]^{2} \leq\left[\left[F_{1}^{2}\right]\right]\left[\left[F_{2}^{2}\right]\right]
$$

Choosing $F_{2}=O_{k}$, we get that for every $k$-labeled graph $F$,

$$
\left[\left[F^{2}\right]\right] \geq[[F]]^{2} \geq 0
$$

Let $F^{\text {sub }}$ denote the subdivision of graph $F$ obtained by adding one new node on each edge.

Lemma 3.5 If $F \leq G$, then $F^{\text {sub }} \leq G^{\text {sub }}$.

Proof. For every $U \in \mathcal{W}, t\left(F^{\text {sub }}, U\right)=t\left(F, U \circ U^{\top}\right) \leq t\left(G, U \circ U^{\top}\right)=t\left(G^{\text {sub }}, U\right)$.

The next lemma will be the workhorse throughout this paper.

Lemma 3.6 Let $F$ be an (unlabeled) bigraph, let $S \subseteq V(F)$, and let $H_{1}, \ldots, H_{m}$ be the connected components of $F \backslash S$. Assume that each node in $S$ has neighbors in at most two of the $H_{i}$. Let $F_{i}$ denote the graph consisting of $H_{i}$, its neighbors in $S$, and the edges between $H_{i}$ and $S$. Let us label the nodes of $S$ in every $F_{i}$. Then

$$
F^{2} \leq \prod_{i=1}^{m}\left[\left[F_{i}^{2}\right]\right] .
$$

Proof. Let $F_{0}$ denote the subgraph induced by $S$, and consider the nodes of $F_{0}$ labeled $1, \ldots, k$; we may assume that these nodes are labeled the same way in every $F_{i}$. Then using that $\left|t_{x_{1} \ldots x_{k}}\left(F_{0}, U\right)\right| \leq 1$, we get

$$
\begin{aligned}
|t(F, U)| & =\left|\int_{I^{k}} \prod_{i=0}^{m} t_{x_{1} \ldots x_{k}}\left(F_{i}, U\right) d x_{1} \ldots d x_{k}\right| \\
& \leq \int_{I^{k}} \prod_{i=1}^{m}\left|t_{x_{1} \ldots x_{k}}\left(F_{i}, U\right)\right| d x_{1} \ldots d x_{k} .
\end{aligned}
$$

Hence Lemma 3.3 implies the assertion.

As a special case, we see that if $F$ contains two nonadjacent nodes of degree at least 2 , then $F \leq C_{4}$. More generally,

Corollary 3.7 Let $v_{1}, \ldots, v_{m}$ be independent nodes in an (unlabeled) bigraph $F$ with degrees $d_{1}, \ldots, d_{m}$ such that no node of $F$ is adjacent to more than 2 of them. Then $F^{2} \leq K_{2, d_{1}} \cdots K_{2, d_{m}}$. If $d_{1}, \ldots, d_{m} \geq 2$, then $F^{2} \leq C_{4}^{m}$. 
A hanging path system in a graph $F$ is a set $\left\{P_{1}, \ldots, P_{m}\right\}$ of openly disjoint paths such that the internal nodes of each $P_{i}$ have degree 2 , and at most two of them start at any node. Lemma 3.6 can be used to bound the graph in terms of any hanging path system:

Corollary 3.8 Let $F$ be a bigraph that contains a hanging path system with lengths $r_{1}, \ldots, r_{m}$. Then $F^{2} \leq C_{2 r_{1}} \cdots C_{2 r_{m}}$.

\subsection{Special graphs and examples}

Lemma 3.9 Let $U \in \mathcal{W}_{1}$. Then the sequence $\left(t\left(C_{2 k}, U\right): k=1,2, \ldots\right)$ is nonnegative, logconvex, and monotone decreasing.

With the notation introduced above, we have $C_{2} \geq C_{4} \geq C_{6} \geq \cdots \geq 0$ and $C_{2 k}^{2} \leq$ $C_{2 k-2} C_{2 k+2}$.

Proof. We have $C_{a+b}=\left[\left[P_{a}^{\bullet \bullet} P_{b}^{\bullet \bullet}\right]\right]$. Taking $a=b=k$, nonnegativity follows. Applying inequality (9), we get that $C_{a+b}^{2} \leq C_{2 a} C_{2 b}$. This implies logconvexity. Since the sequence remains bounded by 1 , it follows that it is monotone decreasing.

Monotonicity and logconvexity of the sequence of even cycles imply inequalities between collections of cycles.

Lemma 3.10 Let $1 \leq r_{1} \leq \cdots \leq r_{m}$ and $1 \leq q_{1} \leq \cdots \leq q_{m}$ be integers and assume that $\sum_{i=1}^{j} r_{i} \geq \sum_{i=1}^{j} q_{i}$ for every $1 \leq j \leq m$. Then

$$
C_{2 r_{1}} \cdots C_{2 r_{m}} \leq C_{2 q_{1}} \cdots C_{2 q_{m}}
$$

Proof. We use induction on $m$ and on $r_{1}$. For $m=1$ the assertion is just monotonicity. Let $m \geq 2$. If $r_{1}=q_{1}$, we can delete the first member of each list, and apply induction. If $r_{1}>q_{1}$, then let us replace $r_{1}$ by $r_{1}-1$ and $r_{2}$ by $r_{2}+1$. It is easy to check that the resulting sequence satisfies the conditions of the Corollary, and so the induction hypothesis applies to it. Furthermore, logconcavity implies that

$$
C_{2 r_{1}} C_{2 r_{2}} \leq C_{2 r_{1}-2} C_{2 r_{2}+2}
$$

and so

$$
C_{2 r_{1}} C_{2 r_{2}} \cdots C_{2 r_{m}} \leq C_{2 r_{1}-2} C_{2 r_{2}+2} \cdots C_{2 r_{m}} \leq C_{2 q_{1}} \cdots C_{2 q_{m}}
$$

As a special case of the last corollary, we get that if $r_{1}, \ldots, r_{m} \geq 1$ and $r=r_{1}+\cdots+r_{m}$, then

$$
C_{2 r_{1}} \cdots C_{2 r_{m}} \leq C_{2}^{m-1} C_{2 r-2 m+2} \leq C_{2 r-2 m+2} .
$$

The following lemma gives an estimate on the product of even cycles which goes in a sense in the opposite direction. 
Lemma 3.11 Let $r_{1}, \ldots, r_{m} \geq 1$ and $r=r_{1}+\cdots+r_{m}$. Then $C_{2 r_{1}} \cdots C_{2 r_{m}} \geq C_{r}^{2}$.

Proof. We split $C_{r}$ into paths of lengths $r_{1}, r_{2}, \ldots, r_{m}$, and apply Lemma 3.6.

Choosing $r_{1}=r_{2}=k$ and $r_{3}=2$ in Lemma 3.11, we get that $C_{2 k+2}^{2} \leq C_{2 k}^{2} C_{4}$. Choosing $m=r-1, r_{1}=\ldots r_{m}=2, q_{1}=\cdots=q_{m-1}=1$ and $q_{m}=r$ in Lemma 3.10, we get that $C_{4}^{r-1} \leq C_{2}^{r-2} C_{2 r}$. Together, these inequalities imply that for every $U \in \mathcal{W}_{1}$, the density $t\left(C_{2 k}, U\right)$ tends to 0 exponentially with $k$ (unless $W=1$ almost everywhere):

Corollary 3.12 For all $r \geq 2, C_{4}^{r-1} \leq C_{2 r} \leq C_{4}^{r / 2}$.

The value of a hanging path system is the total number of their internal nodes. We get by Corollary 3.8 and Lemma 3.10.

Corollary 3.13 Let $F$ be a simple bigraph that contains a hanging path system with path lengths at most $r$ and value at least $2 r-2$. Then $F \leq C_{2 r}$. If the value is larger than $2 r-2$, then $F^{2} \leq C_{2 r}^{2} C_{4}$.

We can get similar inequalities for paths, of which we only state two, which will be needed. Recall that $P_{n}$ denotes the path with $n$ nodes and $n-1$ edges.

Lemma 3.14 For all $a, b \geq 1$, we have

(a) $P_{a+b+1}^{2} \leq P_{2 a+1} P_{2 b+1}$;

(b) $P_{2 a+b+1}^{4} \leq P_{2 a+1}^{4} C_{4 b}$.

Proof. Since $P_{a+b+1}=\left[\left[P_{a+1}^{\bullet} P_{b+1}^{\bullet}\right]\right]$, the first inequality follows by (9). To get the second, we use the first to get

$$
P_{2 a+b+1}^{2} \leq P_{2 a+1} P_{2 a+2 b+1} .
$$

Cut $P_{2 a+2 b+1}$ into pieces $P_{a+1}^{\bullet}, P_{2 b+1}^{\bullet \bullet}$ and $P_{a+1}^{\bullet}$, and apply Lemma 3.6, we get

$$
P_{2 a+2 b+1}^{2} \leq P_{2 a+1}^{2} C_{4 b}
$$

and hence

$$
P_{2 a+b+1}^{4} \leq P_{2 a+1}^{2}\left(P_{2 a+1}^{2} C_{4 b}\right)=P_{2 a+1}^{4} C_{4 b}
$$

The densities of complete bigraphs in graphons have similar, but also quite different properties to cycle densities. We start with the similarity.

Lemma 3.15 Let $U \in \mathcal{W}_{1}$. Then for every $h \geq 1$, the sequence $\left(t\left(K_{h, 2 k}, U\right): k=\right.$ $1,2, \ldots)$ is nonnegative, logconvex and monotone decreasing. 
Proof. The proof is similar to the proof of Lemma 3.9, based on the equation $K_{h, a+b}=$ $\left[\left[K_{h, a}^{\bullet} K_{h, b}^{\bullet}\right]\right]$.

For complete bigraphs, however, we don't have a bound similar to Corollary 3.12 (see Example 1). But we do have the following inequality.

Lemma 3.16 For all $n \geq 3$, we have $K_{n, n}^{2} \leq K_{2, n}^{2} C_{2}$.

Proof. Let $H$ be the 2-labeled graph obtained from $K_{n, n}$ by deleting an edge and labeling its endpoints. Then $K_{n, n}=\left[\left[K_{2}^{\bullet \bullet} H\right]\right]$, and hence

$$
K_{n, n}^{2} \leq\left[\left[\left(K_{2}^{\bullet \bullet}\right)^{2}\right]\right]\left[\left[H^{2}\right]\right]=C_{2}\left[\left[H^{2}\right]\right] .
$$

Now taking two unlabeled nodes from one color class from one copy of $H$ and two unlabeled nodes from the other color class from the other copy, we get a set of 4 independent nodes of degree $n$ such that no three have a neighbor in common. Hence Corollary 3.7 implies that $\left[\left[H^{2}\right]\right] \leq K_{2, n}^{2}$, which proves the lemma.

Example 1 Let $U:[0,1]^{2} \rightarrow[-1,1]$ be defined by

$$
U(x, y)= \begin{cases}-1, & \text { if } x, y \geq 1 / 2 \\ 1 & \text { otherwise. }\end{cases}
$$

Then it is easy to calculate that for all $n, m \geq 1, t\left(K_{n, m}, U\right)=\frac{1}{2}$.

We conclude with two inequalities that bound subgraph densities with prescribed images for the labeled nodes.

Lemma 3.17 For all $U \in \mathcal{W}, x \in I$ and $r \geq 2$,

$$
0 \leq t_{x}\left(C_{2 r}^{\bullet}, U\right) \leq t\left(C_{4 r-4}, U\right)^{1 / 2}
$$

Proof. The first inequality follows from the formula

$$
t_{x}\left(C_{2 r}^{\bullet}, U\right)=\int_{I} t_{u x}\left(P_{r+1}^{\bullet \bullet}, U\right)^{2} d u .
$$

For the second, write

$$
t_{x}\left(C_{2 r}^{\bullet}, U\right)=\int_{I^{2}} U(x, u) t_{u v}\left(P_{2 r-1}^{\bullet \bullet}, U\right) U(v, x) d u d v,
$$

and apply the Cauchy-Schwarz inequality:

$$
\begin{aligned}
t_{x}\left(C_{2 r}^{\bullet}, U\right)^{2} & \leq \int_{I^{2}} U(x, u)^{2} U(v, x)^{2} d u d v \int_{I^{2}} t_{u v}\left(P_{2 r-1}^{\bullet \bullet}, U\right)^{2} d u d v \\
& =t_{x}\left(C_{2}^{\bullet}, U\right)^{2} t\left(C_{4 r-4}, U\right) \leq t\left(C_{4 r-4}, U\right) .
\end{aligned}
$$


Lemma 3.18 For all $U \in \mathcal{W}, k \geq 4$ and $x, y \in I$,

$$
\left|t_{x y}\left(P_{k}^{\bullet \bullet}, U\right)\right| \leq t\left(C_{4 k-12}, U\right)^{1 / 4} .
$$

Proof. We can write

$$
t_{x y}\left(P_{k}^{\bullet \bullet}, U\right)=\int U(x, u) t_{u y}\left(P_{k-1}^{\bullet \bullet}, U\right) d u .
$$

Hence by the Cauchy-Schwarz inequality,

$$
\begin{aligned}
t_{x y}\left(P_{k}^{\bullet \bullet}, U\right)^{2} & \leq \int U(x, u)^{2} d u \int t_{u y}\left(P_{k-1}^{\bullet \bullet}, U\right)^{2} d u \\
& \leq \int t_{u y}\left(P_{k-1}^{\bullet \bullet}, U\right)^{2} d u=t_{y}\left(C_{2 k-2}^{\bullet}, U\right) d u .
\end{aligned}
$$

Applying Lemma 3.17 the proof follows.

\subsection{The main inequalities between graphs}

Our main lemma is the following.

Lemma 3.19 Let $F$ be a bigraph with all degrees at least 2, with girth $2 r$, which is not a single cycle or a complete bigraph. Then $F \leq C_{2 r} C_{4}^{1 / 4}$.

Before proving this lemma, we need some preparation. Let $T$ be a rooted tree. By its min-depth we mean the minimum distance of any leaf from the root. (As usual, the depth of $T$ is the maximum distance of any leaf from the root.)

Lemma 3.20 Let $T$ be a rooted tree with min-depth $h$ and depth $g$, with its leaves labeled. Then $\left[\left[T^{2}\right]\right]$ contains a hanging path system with value at least $g+\max (0, h-3)$, in which the paths are not longer than $\max (g, 2)$.

Proof. The proof is by induction on $|V(T)|$. We may assume that the root has degree 1 , else we can delete all branches but the deepest from the root. Let $a$ denote the length of the path $P$ in $T$ from the root $r$ to the first branching point or leaf $v$.

If $P$ ends at a leaf, then the whole tree is a path of length $a=g=h$. If $a=1$, we get a hanging path in $\left[\left[T^{2}\right]\right]$ of length 2 , and so of value $1=1+\max (0,-1)$. If $a \geq 2$, then we can even cut this into two, and get two hanging paths in $\left[\left[T^{2}\right]\right]$ of length $a$, which has value $2 a-2 \geq a+\max (0, a-3)$.

If $P$ ends at a branching point, then we consider two subtrees $F_{1}, F_{2}$ rooted at $v$ (there may be more), where $F_{1}$ has depth $g-a$. Clearly, $F_{1}$ has min-depth at least $h-a$ and $F_{2}$ has min-depth and depth at least $h-a$. By induction, $\left[\left[F_{1}^{2}\right]\right]$ and $\left[\left[F_{2}^{2}\right]\right]$ contain hanging path systems of value $g-a+\max (0,(h-a)-3)$ and $h-a+\max (0,(h-a)-3)$, respectively. 
The two systems together have value at least $g+h-2 a$, and they form a valid system since $v$ (and its mirror image) are contained in at most one path of each system. If $a=1$, we are done, since clearly $h \geq 2$ and so $g+h-2 \geq g+\max (0, h-3)$.

Assume that $a \geq 2$. Let $F_{3}$ be obtained from $F_{2}$ by deleting its root. By induction, $\left[\left[F_{1}^{2}\right]\right]$ contains hanging path systems of value $g-a+\max (0, h-a-3)$, and $\left[\left[F_{3}^{2}\right]\right]$ contains a hanging path system of value $h-a+\max (0, h-a-4)$. We can add $P$ and its mirror image, to get a hanging path system of value

$$
\begin{gathered}
(g-a)+(h-a-1)+\max (0, h-a-3)+\max (0, h-a-4)+2(a-1) \\
\geq(g-a)+(h-a-1)+2(a-1)=g+h-3=g+\max (0, h-3),
\end{gathered}
$$

since $h \geq a+1 \geq 3$. We know that every path constructed lies in the tree or in its mirror image, except for the paths in the case $g=1$. In the case $g \geq 2$, the length of these paths is at most $g$, in the case $g=1$, their length is 2 .

Proof of Lemma 3.19. We distinguish several cases.

Case 1. $r=2$. By hypothesis, $F$ is not a complete bigraph, and hence we can choose nonadjacent nodes $u$ and $v$ from different bipartition classes. Let $N$ denote the set of neighbors of $u,|N|=d$, and let $F_{0}$ denote the graph $F-u$ with the neighbors of $u$ labeled. Then $F \cong\left[\left[F_{0} K_{d, 1}^{\bullet}\right]\right]$, and hence by (12),

$$
F^{2} \leq\left[\left[F_{0}^{2}\right]\right] \cdot\left[\left[\left(K_{d, 1}^{\bullet}\right)^{2}\right]\right]=\left[\left[F_{0}^{2}\right]\right]\left[\left[K_{d, 2}\right]\right] \leq\left[\left[F_{0}^{2}\right]\right] C_{4} .
$$

Now let $v_{1}$ and $v_{2}$ be the two copies of $v$ in $F_{0}^{2}$, and $w$, any third node in the same bipartition class. These three nodes have no neighbor in common, so by Corollary 3.7 we get that $\left[\left[F_{0}^{2}\right]\right] \leq C_{4}^{3 / 2}$, and so $F \leq C_{4}^{5 / 4}$.

Case 2. $F$ is disconnected. If one of the components is not a single cycle, we can replace $F$ by this component. If $F$ is the disjoint union of single cycles $C_{2 r_{1}}, \ldots, C_{2 r_{k}}$ $(k \geq 2)$, then $F=C_{2 r_{1}} \cdots C_{2 r_{k}} \leq C_{2 r}^{k} \leq C_{2 r} C_{4}$.

So we may assume that $F$ is connected. Then it must have at least one node of degree larger than 2.

Case 3. $F$ has at most one node of degree larger than 2 in each color class. Let $u_{1}$ and $u_{2}$ be two nodes, one in each color class, such that all the other nodes have degree 2. Then $F$ must consist of one or more odd paths connecting $u_{1}$ and $u_{2}$, and even cycles attached at $u_{1}$ and/or $u_{2}$.

If there is an even cycle attached at (say) $u_{1}$, then this cycle gives a hanging path system consisting of 2 paths of length $r$, and we can add a third path of length 2 starting at $u_{2}$ but not reaching $u_{1}$. So by Lemma 3.6. $F \leq C_{2 r} \leq C_{2 r} C_{4}^{1 / 2}$.

So we may assume that $F$ consists of openly disjoint paths connecting $u_{1}$ and $u_{2}$. Since $F$ is not a single cycle, there are at least three paths. Let $a_{1} \leq a_{2} \leq a_{3}$ be their lengths. Clearly $a_{1}+a_{2} \geq 2 r$. If $a_{2} \geq r+1$, then we have two hanging paths of length $r+1$, which 
implies that $F \leq C_{2 r+2} \leq C_{2 r} C_{4}^{1 / 2}$. So we may assume that $a_{1}=a_{2}=r$. If $a_{3} \geq 4$, then we can select two paths of length $r$ and path of length 2 disjoint from them, which gives $F \leq C_{2 r} C_{4}^{1 / 2}$.

So we get to the special case when $F$ consists of 3 or more paths of length 3 connecting $u_{1}$ and $u_{2}$. In this case, we use Lemma 3.18.

$$
\begin{aligned}
t(F, U) & =\int_{I^{2}} t_{x y}\left(P_{4}^{\bullet \bullet}, U\right)^{3} d x d y \leq t\left(C_{4}, U\right)^{1 / 4} \int_{I^{2}} t_{x y}\left(P_{4}^{\bullet \bullet}, U\right)^{2} d x d y \\
& =t\left(C_{6}, U\right) t\left(C_{4}, U\right)^{1 / 4}
\end{aligned}
$$

Case 4. Suppose that there are two nodes $u_{1}, u_{2}$ in the same bipartition class of $F$ of degree at least 3 .

Let $S_{1}$ be the set of nodes $x$ in $F$ with $d\left(x, u_{1}\right) \leq \min \left(r-2, d\left(x, u_{2}\right)-2\right)$, and let $S_{1}^{\prime}=N\left(S_{1}\right) \backslash S_{1}$. We define $S_{2}$ and $S_{2}^{\prime}$ analogously. Let $F_{i}$ be the subgraph induced by $S_{i} \cup S_{i}^{\prime}$. Consider the nodes of $F_{i}$ in $S_{i}^{\prime}$ labeled. Lemma 3.6 implies that

$$
F^{2} \leq F_{1}^{2} F_{2}^{2}
$$

Hence to complete the proof, it suffices to show that $F_{1}^{2} \leq C_{2 r} C_{4}^{1 / 4}$ and $F_{1}^{2} \leq C_{2 r}$, or the other way around. This will follow by Corollary 3.13. if we construct in $F_{1}^{2}$ a hanging path system of paths of length at most $r$ with value $2 r-1$ and in $F_{2}^{2}$, a hanging path system of paths of length at most $r$ with value $2 r-2$ (or the other way around).

Claim 1 The subgraph $F_{i}$ is a tree with leaf set $S_{i}^{\prime}$. Every $x \in S_{i}^{\prime}$ satisfies $d\left(x, u_{1}\right)=$ $\min \left(r-1, d\left(x, u_{2}\right)\right)$.

From the fact that $F$ has girth $2 r$ it follows that $F_{i}$ is a tree. The nodes in $S_{i}$ are not endnodes of $F_{i}$, since their degree in $F$ is at least 2 and all their neighbors are nodes of $F_{i}$. It is also trivial that the nodes in $S_{i}^{\prime}$ are endnodes. Let $x \in S_{i}^{\prime}$, then $x \notin S_{i}$ and hence $d\left(x, u_{1}\right) \geq \min \left(r-1, d\left(x, u_{2}\right)-1\right)$. But $d\left(x, u_{1}\right)$ and $d\left(x, u_{2}\right)$ have the same parity, and hence it follows that $d\left(x, u_{1}\right) \geq \min \left(r-1, d\left(x, u_{2}\right)\right)$. On the other hand, $x$ has a neighbor $y \in S_{i}$, and hence $d\left(x, u_{1}\right) \leq d\left(y, u_{1}\right)+1 \leq r-1$, and $d\left(x, u_{1}\right) \leq d\left(y, u_{1}\right)+1 \leq$ $d\left(y, u_{2}\right)-1 \leq d\left(x, u_{2}\right)$. This implies that $d\left(x, u_{1}\right) \leq \min \left(r-1, d\left(x, u_{2}\right)\right)$, which proves the claim.

Claim 2 There is no edge between $S_{1}$ and $S_{2}$.

Indeed, suppose that $x_{1} x_{2}$ is such an edge, $x_{i} \in S_{i}$. Then $d\left(x_{1}, u_{1}\right)<d\left(x_{2}, u_{2}\right)$, which by parity means that $d\left(x_{1}, u_{1}\right) \leq d\left(x_{2}, u_{2}\right)-2$. But then $d\left(x_{2}, u_{1}\right) \leq d\left(x_{1}, u_{1}\right)+1 \leq$ $d\left(x_{2}, u_{2}\right)-1 \leq d\left(x_{2}, u_{1}\right)$, showing that $x_{2} \notin S_{2}$.

Claim 3 Let $y \neq x$ be two leaves of $F_{1}$. Then $d(r, x)+d(r, y)+d(x, y) \geq 2 r$. 
If $d(r, x)=r-1$ or $d(r, y)=r-1$ then this is trivial, so suppose that $d(r, x), d(r, y) \leq$ $r-2$. Then by Claim 1, we must have $d\left(x, u_{2}\right)=d\left(x, u_{1}\right)$ and $d\left(y, u_{2}\right)=d\left(y, u_{1}\right)$. Going from $x$ to $u_{2}$ to $y$ and back to $x$ in $F$, we get a closed walk of length $d(r, x)+d(r, y)+d(x, y)$, which contains a cycle of length no more than that, which implies the inequality in the Claim.

To construct the hanging path systems in $F_{1}^{2}$ and $F_{2}^{2}$, we need to distinguish two cases.

Case 4a. All branches of $F_{1}$ are single paths. Let $a_{1} \leq \cdots \leq a_{d}$ be their lengths. Claim 3 implies that $a_{1}+a_{2} \geq r$, so $a_{2} \geq r / 2$. The graph $F_{1}^{2}$ consists of paths $Q_{1}, \ldots, Q_{d}$ of length $2 a_{1}, \ldots, 2 a_{d}$ connecting $u_{1}$ and its mirror image $u_{1}^{\prime}$. Select subpaths of length $r$ from $Q_{2}$ and $Q_{3}$, this gives a hanging path system of value $2 r-2$. If $a_{1} \geq 2$, then we can add to this a path of length 2 from $Q_{1}$ not containing its endpoints, and we get a path system of value $2 r-1$. So we may assume that $a_{1}=1$. Then $a_{2} \geq r-1>r / 2$, and so $2 a_{2}, 2 a_{r}>r$. Thus we can select the paths of length $r$ from $Q_{2}$ and $Q_{3}$ so that one of them misses $u_{1}$ and the other one misses $u_{1}^{\prime}$. The we can add $Q_{1}$ to the system, and conclude as before.

Case $4 \mathrm{~b}$. At least one of the branches of $F_{1}$, say $A$, is not a single path. Let $a$ be the length of the path $Q$ from the root $u_{1}$ to the first branch point $v$. Let $T_{1}, T_{2}$ be two subtrees of $A$ rooted at $v$, of depth $d_{1}$ and $d_{2}$. Let $B$ and $C$ be two further branches, of depth $b$ and $c$, respectively, where $b \geq c$. By Claim [3] we have $d_{1}+d_{2}+a \geq r$ and $b+c \geq r$.

If $a=1$, then we choose a hanging path system from $T_{1}^{2}$ of value $d_{1}$, from $T_{2}^{2}$ of value $d_{2}$, from $B^{2}$ of value $b$ and from $C^{2}$ of value $c$. This is a total of $d_{1}+d_{2}+b+c \geq 2 r-1$.

If $a \geq 2$, then we choose a hanging path system from $T_{1}^{2}$ of value $d_{1}$, from $\left(T_{2}-v\right)^{2}$ of value $d_{2}-1$, from $B^{2}$ of value $b$ and from $\left(C-u_{1}\right)^{2}$ of value $c-1$. Leaving out $v$ from $T_{2}$ and $u_{1}$ from $C$ allows us to add $Q$ and its mirror image of value $2(a-1)$. This is a total value of

$$
d_{1}+d_{2}-1+2(a-1)+b+c-1 \geq 2 r+a-4 \geq 2 r-2 .
$$

If equality holds in all estimates, then $d_{1}+d_{2}+a=r, b+c=r$, and $a=2$. It also follows that $b \leq 3$, or else we get a larger system in $B$. Note that the depth of $A$ is at least $a+1=3$, and $c \leq r / 2 \leq b \leq 3$.

If $B$ is a single path, then we can select a hanging path of length $r$ from $B^{2}$, of value $r-1>b-1$, and we have gained 1 relative to the previous construction. So we may assume that $B$ is not a single path. Then applying the same argument as above with $A$ and $B$ interchanged, we get that $b=3$, and the depth of $A$ is also 3 . Hence $d_{1}=d_{2}=1$ and $r=d_{1}+d_{2}+a=4$. It follows that $c=r-b=1$, so $C$ consists of a single edge.

If $u_{1}$ has degree larger than 3 , then applying the argument to $A, B$ and a fourth branch $D$, we get that $D$ must have depth 1 , but this contradicts Claim 3 . Hence the degree of $u_{1}$ is 3 .

If $A$ has at least 3 leaves, then these must be connected to $u_{2}$ by disjoint paths of length 3. Since $u_{2}$ must be connected to the endpoint of $C$ as well by Claim 1, we get 
that $u_{2}$ has degree at least 4 , and so $F_{2} \geq C_{2 r} C_{4}^{1 / 2}$.

So $A$ and similarly $B$ have two leaves, and $F_{1}$ is a 10 -node tree consisting of a path with 5 nodes and 2 endnodes hanging from its endnodes and 1 from its middle node. $F_{2}$ must be the same, or else we are done. There is only one way to glue two copies of this tree together at their endnodes to get a graph of girth 8 , and this yields the subdivision of $K_{3,3}$ (by one node on each edge). To settle this single graph, we use that

$$
K_{3,3} \leq C_{2}^{1 / 2} K_{3,2} \leq C_{2}^{1 / 2} C_{4}
$$

by Lemmas 3.16 and 3.15, and so by Lemma 3.5, we have

$$
F=K_{3,3}{ }^{\mathrm{sub}} \leq\left(C_{2}^{\mathrm{sub}}\right)^{1 / 2} C_{4}^{\mathrm{sub}}=C_{4}^{1 / 2} C_{8}
$$

Thus we know that $F_{1}^{2} F_{2}^{2} \leq C_{2 r}$, and for at least one of them $F_{i}^{2} \leq C_{2 r} C_{4}^{1 / 2}$, which implies that $F^{2} \geq F_{1}^{2} F_{2}^{2} \geq C_{2 r} C_{4}^{1 / 4}$.

Lemma 3.6 implies that if $F$ is a bigraph with two nonadjacent nodes $u, v$ of degree 1, then $F \leq P_{3}$. We need a stronger bound:

Lemma 3.21 Let $F$ be a bigraph with two nonadjacent nodes $u, v$ of degree 1 , which is not a star and has at least 3 edges. Then $F \leq P_{3} C_{4}^{1 / 4}$.

Proof. Let $u^{\prime}$ and $v^{\prime}$ denote the neighbors of $u$ and $v$. First, suppose that there is a node $w \neq u, v, u^{\prime}, v^{\prime}$ such that no node is connected to $u, v$ and $w$. If $d(w) \geq 2$, then we can apply Lemma 3.6 to the stars of $u, v$ and $w$, to get $F^{2} \leq P_{3}^{2} K_{2, d(w)} \leq P_{3}^{2} C_{4}$. If $d(w)=1$, then a similar application of Lemma 3.6 gives that $F^{2} \leq P_{3}^{3} \leq P_{3}^{2} C_{4}^{1 / 2}$.

Suppose that no such $w$ exists. Then either $F$ is star (which has been excluded), or $F=P_{4}$, and the bound follows from Lemma 3.14(b).

Lemma 3.22 Let $F$ be a bigraph with exactly one node of degree 1 and with girth $2 r$. Then

$$
F \leq \frac{1}{2} C_{2 r} C_{4}^{1 / 8}+\frac{1}{2} P_{3} C_{4}^{1 / 8}
$$

Proof. Let $v$ be the unique node of degree 1 . We can write $F \cong\left[\left[F_{0} P_{2}^{\bullet}\right]\right]$, where $F_{0}$ is a 1-labeled graph in which all nodes except possibly the labeled node $v$ have degree at least 2. By (12), we get that $F^{2} \leq\left[\left[\left(P_{2}^{\bullet}\right)^{2}\right]\right]\left[\left[F_{0}^{2}\right]\right] \cong P_{3}\left[\left[F_{0}^{2}\right]\right]$. Here $\left[\left[F_{0}^{2}\right]\right]$ is a graph with girth $2 r$ and all degrees at least 2, which is clearly neither a cycle nor a complete bipartite graph. Hence by Lemma 3.19, we get $F^{2} \leq P_{3} C_{2 r} C_{4}^{1 / 4}$. Thus

$$
|t(F, U)| \leq \sqrt{t\left(P_{3}, U\right) t\left(C_{2 r}, U\right)} t\left(C_{4}, U\right)^{1 / 8} \leq \frac{1}{2}\left(t\left(C_{2 r}, U\right)+t\left(P_{3}, U\right)\right) t\left(C_{4}, U\right)^{1 / 8} .
$$




\section{Local Sidorenko Conjecture}

The Sidorenko Conjecture asserts that $t(F, W)$ is minimized by the function $W \equiv 1$ among all functions $W \geq 0$ with $\int W=1$. The following theorem asserts that this is true at least locally.

Theorem 4.1 Let $F$ be a simple bigraph with $m$ edges. Let $W \in \mathcal{W}$ with $\int W=1$, $0 \leq W \leq 2$ and $\|W-1\|_{\square} \leq 2^{-8 m-2}$. Then $t(F, W) \geq 1$.

Proof. Using (5), it suffices to prove the result under a slightly weaker condition $t\left(C_{4}, W-1\right) \leq 2^{-8 m}$. We may assume that $F=(V, E)$ is connected, since otherwise, the argument can be applied to each component. Let $U=W-1$, then we have the expansion

$$
t(F, W)=\sum_{F^{\prime}} t\left(F^{\prime}, U\right)
$$

where $F^{\prime}$ ranges over all spanning subgraphs of $F$. Since isolated nodes can be ignored, we may instead sum over all subgraphs with no isolated nodes (including the term $F^{\prime}=K_{0}$, the empty graph). One term is $t\left(K_{0}, U\right)=1$, and every term containing a component isomorphic to $K_{2}$ is 0 since $t\left(K_{2}, U\right)=\int U=0$.

Based on (10), we can identify two special kinds of nonnegative terms in (14), corresponding to copies of $P_{3}$ and to cycles in $F$. We show that the remaining terms do not cancel these, by grouping them appropriately.

(a) For each node $i \in V$, let $\sum_{\nabla(i)}$ denote summation over all subgraphs $F^{\prime}$ with at least two edges that consist of edges incident with $i$. Let $d_{i}$ denote the degree of $i$ in $F$, assume that $d_{i} \geq 2$, and set $t(x)=t_{x}\left(K_{2}^{\bullet}, U\right)$. Then using that $t(x) \geq-1$ and Bernoulli's Inequality,

$$
\begin{aligned}
\sum_{\nabla(i)} t\left(F^{\prime}, U\right) & =\int_{I} \sum_{k=2}^{d_{i}}\left(\begin{array}{c}
d_{i} \\
k
\end{array}\right) t(x)^{k} d x=\int_{I}(1+t(x))^{d_{i}}-1-d_{i} t(x) d x \\
& \geq \int_{I}(1+t(x))\left(1+\left(d_{i}-1\right) t(x)\right)-1-d_{i} t(x) d x \\
& =\int_{I}\left(d_{i}-1\right) t(x)^{2} d x=\left(d_{i}-1\right) t\left(P_{3}, U\right) .
\end{aligned}
$$

Hence the terms in (14) that correspond to stars sum to at least

$$
\sum_{\text {stars }} t\left(F^{\prime}, U\right) \geq \sum_{i}\left(d_{i}-1\right) t\left(P_{3}, U\right)=(2 m-n) t\left(P_{3}, U\right) .
$$

(b) Another special sum we consider consists of complete bigraphs that are not stars. Fixing a subset $A$ with $|A| \geq 2$ in the first bipartition class of $F$ with $h \geq 2$ common 
neighbors, and fixing the variables in $A$, the sum over such complete bigraphs with $A$ as one of the bipartition classes is

$$
\sum_{j=2}^{h}\left(\begin{array}{l}
h \\
j
\end{array}\right)\left(\int_{I} \prod_{i \in A} U\left(x_{i}, y\right) d y\right)^{j} \geq(h-1)\left(\int_{I} \prod_{i \in A} U\left(x_{i}, y\right) d y\right)^{2}
$$

by the same computation as above. This gives that this sum is nonnegative.

(c) Next, consider those terms $F^{\prime}$ with at least two endnodes that are not stars. For such a term we have

$$
\left|t\left(F^{\prime}, U\right)\right| \leq t\left(P_{3}, U\right) t\left(C_{4}, U\right)^{1 / 4} \leq 2^{-2 m} t\left(P_{3}, U\right)
$$

(if there are two nonadjacent endpoints, then this follows from Lemma 3.21, else, the left hand side is 0 ). The sum of these terms is, in absolute value, at most

$$
2^{m} 2^{-2 m} t\left(P_{3}, U\right)=2^{-m} t\left(P_{3}, U\right)
$$

(d) If $F^{\prime}$ has all degrees at least 2 and girth $2 r$, and it is not a single cycle or complete bigraph, then $F^{\prime} \leq C_{2 r} C_{4}^{1 / 4}$ by Lemma 3.19, and so

$$
\left|t\left(F^{\prime}, U\right)\right| \leq t\left(C_{2 r}, U\right) t\left(C_{4}, U\right)^{1 / 4} \leq 2^{-2 m} t\left(C_{2 r}, U\right) .
$$

So if we fix $r$ and sum over all such subgraphs, we get, in absolute value, at most

$$
2^{m} 2^{-2 m} t\left(C_{2 r}, U\right)=2^{-m} t\left(C_{2 r}, U\right) \text {. }
$$

(e) Finally, if $F^{\prime}$ has exactly one node of degree 1 and girth $2 r$, then by Lemma 3.22

$$
\left|t\left(F^{\prime}, U\right)\right| \leq \frac{1}{2}\left(t\left(P_{3}, U\right)+t\left(C_{2 r}, U\right)\right) t\left(C_{4}, U\right)^{1 / 8} \leq 2^{-m-1}\left(t\left(P_{3}, U\right)+t\left(C_{2 r}, U\right)\right) .
$$

If we sum over all such subgraphs $F^{\prime}$, then we get less than $t\left(P_{3}, U\right)+\frac{1}{2} \sum_{r \geq 2} t\left(C_{2 r}, U\right)$.

The sum in (a) is sufficient to compensate for the sum in (b) and the first term in (e), while the sum over cycles compensates for the sum in $(d)$ and the second sum in (e). This proves that the total sum in (14) is nonnegative.

\section{Variations}

One can combine the conditions and assume a bound on $\|W-1\|_{\infty}$. It follows from the Theorem that $\|W-1\|_{\infty} \leq 2^{-8 m}$ suffices. Going through the same arguments (in fact, in a somewhat simpler form) we get:

Theorem 5.1 Let $F$ be a simple bigraph with $m$ edges. Let $W \in \mathcal{W}$ with $\int W=1$ and $\|W-1\|_{\infty} \leq 1 /(4 m)$. Then $t(F, W) \geq 1$. 
The condition that $\|W-1\|_{\infty} \leq 1 /(4 m)$ implies trivially that $0 \leq W \leq 2$. It would be interesting to get rid of the condition that $W \leq 2$ under an appropriate bound on $\|W-1\|_{\square}$. In the proof of Theorem 4.1, parts (a) and (b) did not use the upper bound on the values of $W$, but in the rest we could not avoid this. We can only offer the following result.

Theorem 5.2 Let $F$ be a simple bigraph with $m$ edges, let $0<\varepsilon<2^{-1-8 m}$, and let $W \in \mathcal{W}$ such that $W \geq 0, \int W=1,\|W-1\|_{\square} \leq 2^{-1-8 m}$, and $\int_{S \times T} W \leq 2 \lambda(S) \lambda(T)$ whenever $\lambda(S), \lambda(T) \geq 2^{-64 / \varepsilon^{2}}$. Then $t(F, W) \geq 1-\varepsilon$.

Proof. For every function $W \in \mathcal{W}$ and partition $\mathcal{P}=\left\{V_{1}, \ldots, V_{k}\right\}$ of $I$ into a finite number of measurable sets with positive measure, let $W_{\mathcal{P}}$ denote the function obtained by averaging $W$ over the partition classes; more precisely, we define

$$
W_{\mathcal{P}}(x, y)=\frac{1}{\lambda\left(V_{i}\right) \lambda\left(V_{j}\right)} \int_{V_{i} \times V_{j}} W(u, v) d u d v
$$

for $x \in V_{i}$ and $y \in V_{j}$.

The Weak Regularity Lemma of Frieze and Kannan in the form used in [1] implies that there is a partition $\mathcal{P}$ into $K \leq 2^{64 m^{2} / \varepsilon^{2}}$ equal measurable sets such that the function $W_{\mathcal{P}}$ satisfies

$$
\left\|W_{\mathcal{P}}-W\right\|_{\square} \leq \frac{\varepsilon}{4 m}
$$

and hence by the Counting Lemma (Lemma 3.8 in [1]),

$$
\left|t\left(F, W_{\mathcal{P}}\right)-t(F, W)\right| \leq \varepsilon .
$$

Clearly $\int W_{\mathcal{P}}=1, W_{\mathcal{P}} \geq 0$, and for all $x \in V_{i}$ and $y \in V_{j}$,

$$
W_{\mathcal{P}}(x, y)=\frac{1}{\lambda\left(V_{i}\right) \lambda\left(V_{j}\right)} \int_{V_{i} \times V_{j}} W(u, v) d u d v \leq 2 .
$$

Furthermore,

$$
\left\|W_{\mathcal{P}}-1\right\|_{\square} \leq\left\|W_{\mathcal{P}}-W\right\|_{\square}+\|W-1\|_{\square} \leq 2^{-8 m},
$$

Thus Theorem 4.1 implies that $t\left(F, W_{\mathcal{P}}\right) \geq 1$, and hence $t(F, W) \geq t\left(F, W_{\mathcal{P}}\right)-\varepsilon \geq 1-\varepsilon$.

We end with a graph-theoretic consequence of Theorem 5.2 .

Corollary 5.3 Let $F$ be a bigraph with $n$ nodes and $m$ edges, and let $G$ be a graph with $N$ nodes and $M=p\left(\begin{array}{c}N \\ 2\end{array}\right)$ edges. Let $\varepsilon>0$. Assume that $\left|e_{G}(S, T)-p\right| S|| T \| \leq$ $\left(2^{-8 m} p-\varepsilon\right) N^{2}$ for all $S, T \subseteq V(G)$, and $e_{G}(S, T) \leq 2 p|S||T|$ for all $S, T \subseteq V(G)$ with $|S|,|T| \geq 2^{-4 m^{2} / \varepsilon^{2}} N$. Then $t(F, G) \geq p^{m}-\varepsilon$. 
Proof. This follows by applying Theorem 5.2 to the function $W_{G} / p$.

Acknowledgement. My thanks are due to the anonymous referee for suggesting many corrections and improvements.

\section{References}

[1] C. Borgs, J.T. Chayes, L. Lovász, V.T. Sós, and K. Vesztergombi: Convergent Graph Sequences I: Subgraph frequencies, metric properties, and testing, Advances in Math. 219 (2008), 1801-1851.

[2] D. Conlon, J. Fox, B Sudakov: An approximate version of Sidorenko's conjecture, Geom. Func. Anal. 20 (2010), 1354-1366.

[3] P. Erdős and M. Simonovits: Cube-supersaturated graphs and related problems, in: Progress in Graph Theory (ed. J.A. Bondy. U.S.R Murty), Academic Press, (1984), 203-218.

[4] H. Hatami: Graph norms and Sidorenko's conjecture, Israel J. of Math. 175 (2010), $125-150$.

[5] L. Lovász: On the cancellation law among finite relational structures, Periodica Math. Hung. 1 (1971), 145-156.

[6] L. Lovász, B. Szegedy: Limits of dense graph sequences, J. Comb. Theory B 96 (2006), 933-957.

[7] A.F. Sidorenko: Inequalities for functionals generated by bipartite graphs (Russian) Diskret. Mat. 3 (1991), 50-65; translation in Discrete Math. Appl. 2 (1992), 489-504.

[8] A.F. Sidorenko: A correlation inequality for bipartite graphs, Graphs and Combin. 9 (1993), 201-204.

[9] A.F. Sidorenko: Randomness friendly graphs, Random Struc. Alg. 8 (1996), 229-241.

[10] M. Simonovits: Extremal graph problems, degenerate extremal problems, and supersaturated graphs, in: Progress in Graph Theory (ed. J.A. Bondy. U.S.R Murty), Academy Press (1984), 419-437. 